\title{
Review of High-Quality Random Number Generators
}

\author{
Frederick James $^{1}$ D $\cdot$ Lorenzo Moneta $^{1}$
}

Received: 2 March 2019 / Accepted: 7 December 2019 / Published online: 8 January 2020

(c) The Author(s) 2020

\begin{abstract}
This is a review of pseudorandom number generators (RNG's) of the highest quality, suitable for use in the most demanding Monte Carlo calculations. All the RNG's we recommend here are based on the Kolmogorov-Anosov theory of mixing in classical mechanical systems, which guarantees under certain conditions and in certain asymptotic limits, that points on the trajectories of these systems can be used to produce random number sequences of exceptional quality. We outline this theory of mixing and establish criteria for deciding which RNG's are sufficiently good approximations to the ideal mathematical systems that guarantee highest quality. The well-known RANLUX (at highest luxury level) and its recent variant RANLUX++ are seen to meet our criteria, and some of the proposed versions of MIXMAX can be modified easily to meet the same criteria.
\end{abstract}

Keywords Pseudorandom numbers $\cdot$ High quality randomness $\cdot$ Kolmogorov-Anasov mixing $\cdot$ Dynamical chaos · RANLUX $\cdot$ MIXMAX

\section{High-Quality Random Numbers}

We are concerned here with pseudorandom number generators (RNG's), in particular those of the highest quality. It turns out to be difficult to find an operational definition of randomness that can be used to measure the quality of a $\mathrm{RNG}$, that is the degree of independence of the numbers in a given sequence, or to prove that they are indeed independent. The situation for traditional RNG's (not based on Kolmogorov-Anasov mixing) is well described by Knuth in [1]. The book contains a wealth of information about random number generation, but nothing about where the randomness comes from, or how to measure the quality (randomness) of a generator. Now with hindsight, it is not surprising that all the widely-used generators described there were later found to have defects (failing tests of randomness and/or giving incorrect results in Monte Carlo (MC) calculations), with the notable exception of RANLUX, which Knuth does mention briefly in the third edition, but without describing the new theoretical basis.

Frederick James

frederick.james@cern.ch

1 CERN, Geneva, Switzerland

\section{The Need for High Quality}

High-level scientific research, like many other domains, has become dependent on Monte Carlo calculations, both in theory and in all phases of experiments. It is well-known that the MC method is used primarily for calculations that are too difficult or even impossible to perform analytically, so that our science has become dependent to a large extent on the random numbers used in extensive MC calculations. But how do we know if those numbers are random enough? In the early days (1960's) the RNG's were so poor that, even with the very slow computers of that time, their defects were sometimes obvious, and users would have to try a different RNG. When the result looked good, it was assumed to be correct, but we know now that all the generators of that period had serious defects which could give incorrect results not easily detected.

As computers got faster and RNG's got longer periods, the situation evolved quantitatively, but still unacceptable results were occasionally obtained and of course were not published. Until 1992, when the famous paper of Ferrenberg et al. [2] showed that the RNG considered at that time to be the best was giving the wrong answer to a problem in phase transitions, while the older RNG's known to be defective gave the right answer. Since most often we don't have any independent way to know the right answer, it became 
clear that empirical testing of RNG's, at that time the only known way to verify their quality, was not good enough. Fortunately, the particular problem which was detected by Ferrenberg et. al. was soon solved by Martin Lüscher (in [3]), but it became clear that if we were to have confidence in MC calculations, we would need a better way to ensure their quality. Fortunately the theory of Mixing, outlined below, now offers this possibility.

The experience gained from developing, using and discovering defects in many RNG's has taught us some lessons which we summarise here (they are explained in detail in [1]):

1. The period should be much longer than any sequence that will be used in any one calculation, but a long period is not sufficient to ensure lack of defects.

2. Empirical testing can demonstrate that a RNG has defects (if it fails a test), but passing any number of empirical tests can never prove the absence of defects.

3. Making an algorithm more complicated (in particular, combining two or more methods in the same algorithm) may make a better RNG, but it can also make one much worse than a simpler component method alone if the component methods are not statistically independent.

4. It is better to use a RNG which has been studied, whose defects are known and understood, than one which looks good but whose defects are not understood.

5. There is no general method to determine how good a RNG must be for a particular MC application. The best way to ensure that a RNG is good enough for a given application, is to use one designed to be good enough for all applications.

\section{The Theory of Mixing in Classical Mechanical Systems}

It has been known, at least since the time of Poincaré, that classical dynamical systems of sufficient complexity can exhibit chaotic behaviour, and numerous attempts have been made to make use of this "dynamical chaos" to produce random numbers by numerical algorithms which simulate mechanical systems. It turns out to be very difficult to find an approach which produces a practical RNG, fast enough and accurate enough for general MC applications. To our knowledge, only two such attempts have been successful, both based on the same representation and theory of dynamical systems.

This theory grew out of the study of the asymptotic behaviour of classical mechanical systems that have no analytic solutions, developed largely in the Soviet Union around the middle of the twentieth century by Kolmogorov, Rokhlin, Anosov, Arnold, Sinai and others. See, for example, Arnold and Avez [4] for the theory. At the time, these mathematicians were certainly not thinking of RNG's, but it turns out that their results can be used to produce sequences of random numbers that have some of the properties of the trajectories of the dynamical systems (See Savvidy [5] and Savvidy [6]). The property of interest here is called Mixing, and is usually associated with the names Kolmogorov and Anosov. Mixing is a well-defined concept in the theory, and will be seen to correspond quite exactly to what is usually called independence or randomness.

The representation of dynamical systems appropriate for our purposes is the following:

$x(i+1)=A \times x(i) \bmod 1$,

where $x(i)$ is the $N$-vector of real numbers specifying completely the state of the system at time $i$, and $A$ is a (constant) $N \times N$ matrix which can be thought of as representing the numerical solution to the equations of motion. The N-dimensional state space is a unit hypercube which because of the modulo function becomes topologically equivalent to an $\mathrm{N}$-dimensional torus by identifying opposite faces. The vectors $x$ represent points along the continuous trajectory of the abstract dynamical system in $\mathrm{N}$-dimensional phase space.

All the elements of the matrix $A$ are integers, and the determinant of $A$ must be one. This ensures that $A$ is invertible and the elements of $A^{-1}$ are also integers. The theory is intended for high- (but finite) dimensional systems $(1 \ll N<\infty)$. In practice $N$ will be between 8 and a few hundred.

\section{Mixing and the Ergodic Hierarchy}

Let $x(i)$ and $x(j)$ represent the state of the dynamical system at two times $i$ and $j$. Furthermore, let $v_{1}$ and $v_{2}$ be any two subspaces of the entire allowed space of points $x$, with measures (volumes relative to the total allowed volume), respectively $\mu\left(v_{1}\right)$ and $\mu\left(v_{2}\right)$. Then the dynamical system is said to be a 1-system (with 1-mixing) if

$P\left(x(i) \in v_{1}\right)=\mu\left(v_{1}\right)$

and a 2-system (with 2-mixing) if

$P\left(x(i) \in v_{1}\right.$ and $\left.x(j) \in v_{2}\right)=\mu\left(v_{1}\right) \mu\left(v_{2}\right)$,

for all $i$ and $j$ sufficiently far apart, and for all subspaces $v_{i}$. Similarly, an $n$-system can be defined for all positive integer values $n$. We define a zero-system as having the ergodic property (coverage), namely that the state of the system will asymptotically come arbitrarily close to any point in the state space.

Putting all this together, we have that asymptotically: 
- A system with zero-mixing covers the entire state space.

- A system with one-mixing covers uniformly.

- A system with two-mixing has $2 \times 2$ independence of points.

- A system with three-mixing has $3 \times 3$ independence.

- etc.

Finally, a system with $n$-mixing for arbitrarily large values of $n$ is said to have K-mixing and is a K-system. It is a result of the theory that the degrees of mixing form a hierarchy [7], that is, a system which has $n$-mixing for any value of $n$ also has $i$-mixing for all $i<n$. There are additional systems, in particular Anasov C-systems and Bernoulli B-systems which are also K-systems, but K-systems are sufficient for our purposes.

Now the theory tells us that a dynamical system represented by Eq. (1) will be a K-system if the matrix $A$ has determinant equal to one and eigenvalues $\lambda$, all of which have moduli $\left|\lambda_{i}\right| \neq 1$.

\section{The Eigenvalues of $A$}

We have seen that to obtain K-mixing, none of the eigenvalues of $A$ should lie on the unit circle in the complex plane. In fact, in order to obtain sufficient mixing as early as possible (recall that complete mixing is only an asymptotic property) it is desirable to have the eigenvalues as far as possible from the unit circle.

An important measure of this distance is the Kolmogorov entropy $h$ :

$h=\sum_{k:\left|\lambda_{k}\right|>1} \ln \left|\lambda_{k}\right|$,

where the sum is taken over all eigenvalues with absolute values greater than 1 [It is also equal to the sum over all eigenvalues less than 1 , but then it changes sign]. As its name implies, it is analogous to thermodynamic entropy as it measures the disorder in the system, and it must be positive for an asymptotically chaotic system [This actually follows from the definition if all $|\lambda| \neq 1]$.

Another important measure is the Lyapunov exponent, defined in the following section.

\section{The Divergence of Nearby Trajectories}

The mechanism by which mixing occurs in K-systems can be observed "experimentally" by noting the behaviour of two trajectories which start at nearby points in state space. Using the same matrix $A$, let us start the generator from two different nearby points $a(0)$ and $b(0)$, separated by a very small distance $\delta(a(0), b(0))$. The distance $\delta$ is defined by

$$
\begin{aligned}
\delta(a, b) & =\max _{\kappa} d_{\kappa}, \\
d_{\kappa} & =\min \left\{\left|a_{\kappa}-b_{\kappa}\right|, 1-\left|a_{\kappa}-b_{\kappa}\right|\right\},
\end{aligned}
$$

where $\kappa$ runs over the $N$ components of the vector indicated. Note that the distance defined in this way is a proper distance measure and has the property $0 \leq \delta \leq 1 / 2$. Now we use Eq. (1) on $a(0)$ and $b(0)$ to produce $a(1)$ and $b(1)$, and calculate $\delta(a(1), b(1))$. Then we continue this process to produce two series of points $a(i)$ and $b(i)$, and a set of distances $\delta(i)$, for $i=1,2,3 \ldots$ until the $\delta$ reach a plateau at the value expected for truly random points, which according to Lüscher is $\delta=12 / 25$ for RANLUX. Then it is a well-known result of the theory that, if A represents a K-system, the distances $\delta_{i}$ will diverge exponentially with $i$, so that if plotted on a logarithmic scale, the points $\delta_{i}$ vs. $i$ should lie on a straight line. The inevitable scatter of points should be reduced by averaging the $\delta$ over different starting pairs $(a(0), b(0))$ (the $b(0)$ must of course always be the same very small distance from the $a(0)$, but in different directions).

The rate of divergence of nearby trajectories ( $v$, the Lyapunov exponent) is equal to the logarithm of the modulus of the largest eigenvalue:

$v=\ln |\lambda|_{\max }$,

which, for a K-system, should be the slope of the straight line described above.

\section{Decimation}

The asymptotic independence of $a$ and $b$ is guaranteed by the mixing (if it is a $\mathrm{K}$-system), but as long as $\delta(i)$ remains small, $a(i)$ and $b(i)$ are clearly correlated. The point where the straight line of divergence of nearby trajectories reaches the plateau of constant $\delta$ indicates the number of iterations $m$ required to make the K-system "sufficiently asymptotic", in the sense that the points $a$ and $b$ generated on the following iteration are on average as far apart as independent points would be. We may call this criterion the "2-mixing criterion", since it apparently assures that $2 \times 2$ correlations due to nearby trajectories will be negligible. The question whether this criterion is sufficient to eliminate higher order correlations, is important and will be discussed below in connection with RANLUX++.

Some plots of divergence for real RNG's are given below. If the $\mathrm{K}$-system is used to generate random numbers, to eliminate the correlations due to nearby trajectories, after one vector $a_{i}$ is delivered to the user, the following $m$ vectors $a_{i+1}, a_{i+2}, \ldots a_{i+m}$ must be discarded before the next vector $a_{i+m+1}$ is delivered to the user (decimation). It has become conventional to characterise the degree of decimation by the integer $p$, defined such that after delivering a vector of $N$ random numbers to the user, $p-N$ numbers are skipped before the next $N$ numbers are delivered $[3,8]$. 


\section{From the Theory to the Discrete RNG}

Equation (1) will be used directly to generate random numbers, where $x(0)$ will be the $N$-dimensional seed, and each successive vector $x(i)$ will produce $N$ random numbers. However, in the theory, $x$ is a vector of real numbers, continuous along the unit line. The computer implementation must approximate the real line by discrete rational numbers, which is valid provided the finite period is sufficiently long, and the rational numbers are sufficiently dense, so that the effects of the discreteness are not detectable. Thus the computer implementation has access only to a rational sublattice of the continuous state space, and we must confirm that the discrete approximation preserves the mixing properties of the continuous K-system. Fortunately, the divergence of nearby trajectories offers this possibility, since a theorem usually attributed to Pesin [7] states that a dynamical system has positive Kolmogorov entropy and is, therefore, K-mixing if and only if nearby trajectories diverge exponentially. Then we can expect the discrete system to be K-mixing only if the same condition is satisfied. This is discussed in more detail below in connection with Extended MIXMAX.

\section{The Period}

The most obvious difference between continuous and discrete systems is that continuous systems can have infinitely long trajectories, whereas a computer RNG must always have a finite period. This means that a trajectory 'eats up' state space as it proceeds, since it can never return to a state it has previously occupied without terminating its period. The fact that the available state space becomes progressively smaller indicates necessarily a defect in the RNG, but this defect is easily seen to be undetectable if the period is long enough. According to Maclaren [9], when the period is $P$, using more than $P^{2 / 3}$ numbers from the sequence "leads to excessive uniformity compared to a true random sequence." This is compatible with the RNG folklore which sets the usable limit at $\sqrt{P}$.

\section{Summary of Criteria for Highest Quality}

We will consider as candidates for highest quality RNG's only those based on the theory of chaos in classical mechanical systems, for the simple reason that we know of no other class of systems which can offer-even in some limit which may or may not be attainable - the uniform distribution and lack of correlations guaranteed by k-mixing. To be precise, our criteria are the following:

1. Matrix A must have eigenvalues away from the unit circle, and determinant $=1$.
2. Kolmogorov entropy must be positive (follows from the above).

3. The discrete algorithm must have points sufficiently dense to accurately represent the continuous system.

3. Divergence of nearby trajectories must be exponential (follows from the above) .

4. Decimation must be sufficient to assure that the average distance between successive vectors is the expected distance between independent points.

5. Period must be long enough, $>10^{100}$

6. Some practical criteria: double precision available, portable, repeatable, independent sequences possible.

\section{The High-Quality RNG's: 1. RANLUX}

The first widely-used RNG to offer reliably random numbers was Martin Lüscher's RANLUX, published in 1994. He considered the RNG proposed by Marsaglia and Zaman [10], installed many years ago at CERN with the name RCA RRY, and now known variously as RCARRY, SWB (subtract with borrow) or AWC (add with carry). He discovered that, if the carry bit was neglected (see below), this RNG had a structure that could be represented by Eq. (1), and was, therefore, possibly related to a K-system.

\section{The SWB (RCARRY) Algorithm}

SWB operates on an internal vector of 24 numbers, each with a 24-bit mantissa, and each call to the generator produces one random number which is produced by a single arithmetic operation (addition or subtraction) operating on two of the numbers in the internal vector. Then this random number replaces one of the entries in the internal vector. Lüscher realised that if SWB is called 24 times in succession it generates a 24 -vector which is related to the starting 24-vector by (1), and he could determine the matrix $A$ which would reproduce almost the same sequence as SWB. The only difference would be due to the "carry bit" which is necessary for attaining a long period, but affects only the least significant bit, so it does not alter significantly the mixing properties of the generator. The carry bit is described in detail, both in the paper of Marsaglia and Zaman [10] and that of Lüscher [3].

\section{SWB: The Lyapunov Exponent and Decimation}

The eigenvalues of this "equivalent matrix" were seen to satisfy the conditions for a K-system, but the RNG nevertheless failed several standard tests of randomness. Plotting the evolution of the separation of nearby trajectories immediately indicated the reason for the failure and how to fix it. The 


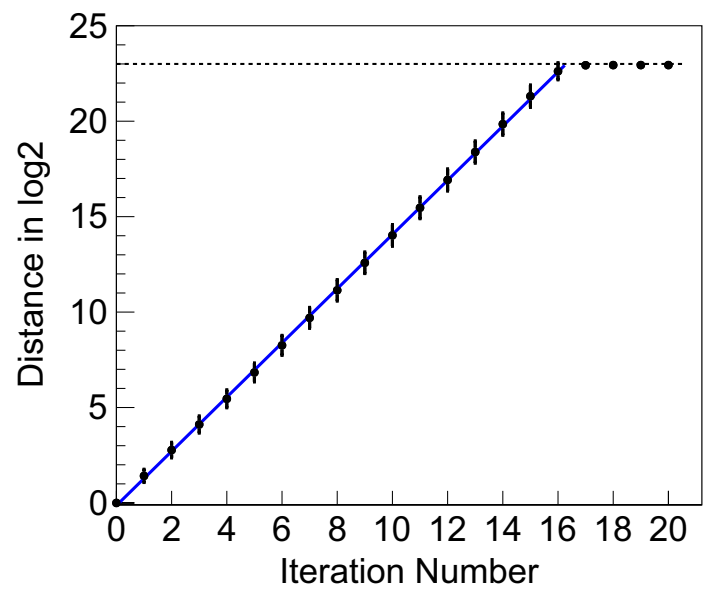

Fig. 1 Divergence of nearby trajectories for RANLUX. The blue line is a straight-line fit to all points

Lyapunov exponent was bigger than one, as required, but not much bigger, so it would require considerable decimation to attain full mixing.

Note that the reason for needing decimation has nothing to do with the carry bit, or even with the discrete approximation to the continuous system. It would in general be needed even for an ideal K-system with continuous $a_{i}$, simply because mixing is only an asymptotic property of K-systems.

The situation is shown in Fig. 1. It can be seen first of all that the first 15 points lie precisely on a straight line, indicating exponential divergence, and therefore, RANLUX has indeed the mixing properties of a K-system. However, we also see that, after generating 24 numbers, it is necessary to throw away (decimate) about $16 \times 24$ numbers before delivering the next 24 numbers to the user, in order that the average separation between nearby trajectories equals the expected separation between independent points in phase space (the 2-mixing criterion). Fortunately, throwing away numbers is typically about twice as fast as delivering them, so the generation time is increased by only about a factor 7 , which is still negligible for many applications, since the basic algorithm is very fast. However, for those users who cannot afford the extra time, RANLUX offers different degrees of decimation, known as "luxury levels". The lowest luxury levels provide very little or no decimation and are very fast; higher levels are slower but more reliable: and the highest level offers sufficient decimation to eliminate the obvious correlation due to nearby trajectories remaining too close. Whether this decimation is sufficient to guarantee the disappearance of all higher order correlations will be discussed below.

\section{RANLUX: The Spectral Test}

The spectral test is not exactly an empirical test, since it cannot be applied to any sequence of numbers, but requires some knowledge of the RNG algorithm [1]. We could, therefore, call it a semi-empirical test which can be used on any linear congruential generator (LCG) with a known multiplier. RNG's in this class have the well-known property that if $d$-tuples of random numbers are used as coordinates of points in a $d$-dimensional hypercube, all the points lie on parallel hyperplanes which are sometimes spaced much more widely than would be the case for independent points. Given the multiplier of the LCG and a value of $d$, the spectral test provides a figure of merit for the spacing of the hyperplanes. This test is a full-period test and is generally considered to be more powerful than the usual empirical tests.

When the first papers on RANLUX were published, Tezuka et al. [11] had already discovered that the algorithm of Marsaglia and Zaman [10] (which was the basis for RANLUX) was in fact equivalent to a linear congruential generator (LCG) with an enormous multiplier. This fact was used by Lüscher [3] to apply the spectral test to RANLUX. He shows that RANLUX passes the test at high luxury levels, but fails at the lower levels as expected.

\section{RANLUX: The Period and the Implementation}

So RANLUX is simply SWB with decimation, where the amount of decimation is a parameter to be chosen by the user: the luxury level. The definitions of the luxury levels may depend on the implementation, so the user should consult local documentation.

The period of SWB is known exactly and is $\approx 5 \times 10^{171}$. This is easily seen to be many orders of magnitude more than all the world's computers could generate in the expected lifetime of the earth, so any defects arising from the finiteness of the state space should remain undetectable.

The original implementation was in Fortran [12] but RANLUX is now used mainly in the implementations in $\mathrm{C}$, available in single precision for 24-bit mantissas, and in double precision for 53-bit mantissas, of which 48 are implemented and the last 5 are zeroes. These are available with documentation from Lüscher's website ( http://lusch er.web.cern.ch/luscher/) and are also installed in several program libraries including CERN and the Gnu Scientific Library. It is part of the $\mathrm{C}++$ standard library. There is also a version [13] using integer arithmetic internally, which may be faster on some platforms. Because of the luxury levels, 
RANLUX is especially suited for testing packages that test RNG's for randomness [14]. If RANLUX passes the test at low luxury levels, then the test is not very sensitive; if it fails at the highest luxury level, then it is likely that the test itself is defective. ${ }^{1}$

\section{The High-Quality RNG's: 2. MIXMAX}

A few years before the publication of RANLUX, George Savvidy et al. in Erevan [5] were working on a different approach to the same problem using the same theory of mixing. Their approach was to look for a family of matrices $A$ which could be defined for any dimension $N$, having eigenvalues far from the unit circle and, therefore, large Lyapunov exponents for different values of $N$, to reduce or even eliminate the need for decimation.

\section{MIXMAX: The Matrix, the Algorithm, and Decimation}

The family of matrices which they found was (for $N \geq 3$ )

$$
A=\left(\begin{array}{ccccccc}
1 & 1 & 1 & 1 & \cdots & 1 & 1 \\
1 & 2 & 1 & 1 & \cdots & 1 & 1 \\
1 & 3+s & 2 & 1 & \cdots & 1 & 1 \\
1 & 4 & 3 & 2 & \cdots & 1 & 1 \\
\vdots & \vdots & \vdots & \vdots & \ddots & \vdots & \vdots \\
1 & N-1 & N-2 & N-3 & \cdots & 2 & 1 \\
1 & N & N-1 & N-2 & \cdots & 3 & 2
\end{array}\right)
$$

where the "magic" integer $s$ is normally zero, but for some values of $N, s=0$ would produce an eigenvalue $\left|\lambda_{i}\right|=1$, in which case a different small integer must be used.

The straightforward evaluation of the matrix-vector product in Eq. (1) requires $O\left(N^{2}\right)$ operations to produce $N$ random numbers, making it hopelessly slow compared with other popular RNG's, so a faster algorithm would be needed. After considerable effort, they reduced the time to $O(N \ln N)$, much faster but still too slow.

George Savvidy's son Konstantin, also a theoretical physicist, found the algorithm [15] that reduced the computation time to $O(N)$, making it competitive in speed with the fastest generators and, very important, the time to generate one number would now be essentially independent of $N$.

Considerable work remained, the most difficult being the determination of the period of the new generator for all interesting values of $N$. The periods are so long that this requires the use of finite (but very large) Galois fields.

By 2014, the essential problems were solved, MIXMAX was being tested at different sites including CERN, and Konstantin Savvidy published the paper [15] giving some of the

\footnotetext{
${ }^{1}$ Several defects have been reported in RANLUX, but in each case it turned out that the test procedure was incorrect or incorrectly applied.
}

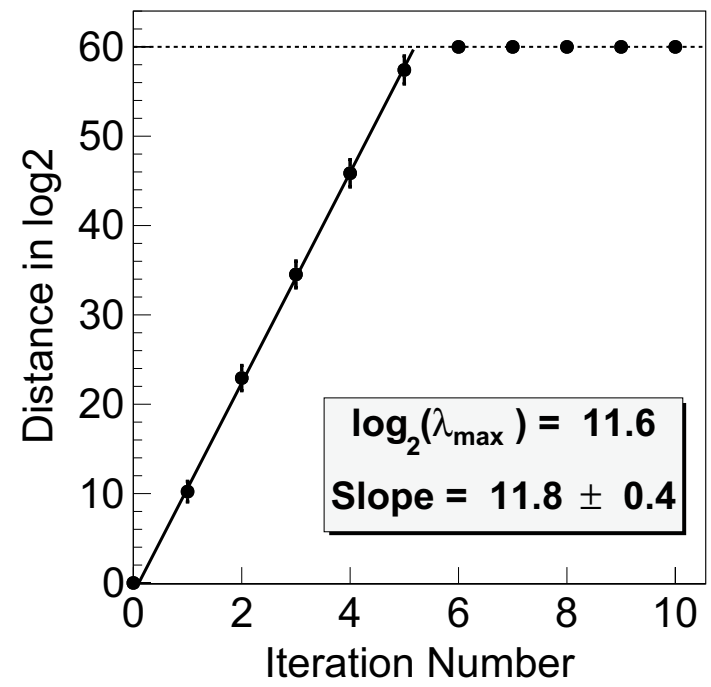

Fig. 2 Divergence of nearby trajectories for MIXMAX 1.0, N = 256

important properties of MIXMAX for 13 different values of the dimension $N$ from 10 to 3150 . The implementation uses 64-bit integer arithmetic internally, so that the user always gets standard double-precision floating-point numbers with 53-bit mantissas. All the periods are longer than $10^{165}$, so that is not a problem. The paper gives the results of the Big Crush test of the TestU01 package [16] for 13 values of $N$, and the test is failed for $N \leq 64^{2}$ but passes for $N \geq 88$. In practice, $N=256$ became the default value, probably because of the results of these tests.

As expected, most of the eigenvalues of the Savvidy matrix are far from the unit circle (compared with those of RANLUX), although there are always a few close to one. For some values of $N$ (including $N=256$ ) the "magic" integer $s$ must be invoked as described above. The moduli of the largest eigenvalues are typically of order $N$. For large $N$, the smallest eigenvalues cluster around $|\lambda|=1 / 4$.

Thus, the Kolmogorov entropy is greater than that of RANLUX, indicating a faster convergence toward the asymptotic independence that is guaranteed by the mixing property. To demonstrate clearly that mixing is indeed occurring, one can plot the divergence of nearby trajectories as was done for RANLUX, and verify that the divergence is exponential as it should be for a continuous $\mathrm{K}$-system. Then the same plot can be used to determine how much (if any) decimation is required to eliminate the correlation of nearby trajectories.

\footnotetext{
2 for $N=64$, the failure of the Big Crush test is termed "only marginal", but the probability of $\chi^{2} \geq 372$ for 232 degrees of freedom is $1.12 \times 10^{-8}$
} 


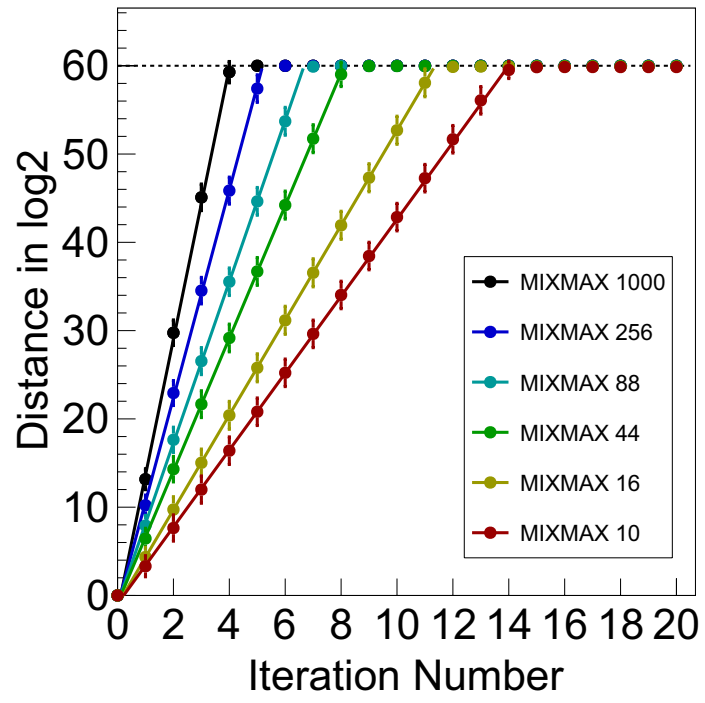

Fig. 3 Divergence of nearby trajectories for MIXMAX 1.0, $N=10$, $16,44,88,256,1000$

Unfortunately, there was nothing in the published paper [15] on the divergence of nearby trajectories and no mention of decimation. At CERN, we were mainly interested in getting a RNG of the highest quality with no known defects, so we did our own calculation of the divergence of nearby trajectories for $N=256$ as shown in Fig. 2. It can be seen that (1) the trajectories do indeed diverge exponentially and (2) after five iterations, the average distance between two nearby trajectories has almost reached the asymptotic value (slightly less than the maximum value of $1 / 2$ ). Note that as long as the average distance between trajectories is significantly less than the asymptotic value, this is direct evidence of a defect, since it indicates that the trajectories still "remember, where they came from", which would not be the case if the points were independent. But this generator passed the Big Crush test, so we see clearly that the divergence of trajectories is more sensitive to this defect than Big Crush.

Therefore, MIXMAX 256 requires decimation, but less than RANLUX, making it a candidate for the world's fastest high-quality RNG. This version was introduced at CERN as a standard generator for ROOT, with the possibility to choose different levels of decimation.

We have also considered the properties of MIXMAX for other values of the dimension $N$. In particular, we have looked at the divergence of nearby trajectories for some values of $N$ for which the period was published in [15] . These curves are shown in Fig. 3 for $N=10,16,44,88$, 256 and 1000. (We have also tried $N=44000$, but the overheads involved in handling such a large matrix are not worth the possible gains.) It is seen that for all these values of $N$, the divergence is remarkably exponential, not only for small separations as required by the theory, but for
Table 1 Number of iterations of decimation required for full separation with original MIXMAX for different values of $N$

\begin{tabular}{ll}
\hline MIXMAX $N$ & Decimation \\
\hline 10 & 14 \\
16 & 11 \\
44 & 8 \\
88 & 6 \\
256 & 5 \\
1000 & 4 \\
\hline
\end{tabular}

all separations right up to the expected asymptotic value which is always close to $1 / 2$. In addition, the slope of the exponential is in all cases equal to the maximum Lyapunov exponent as predicted by the theory. And since the Lyapunov exponent increases with $N$, the decimation required for maximum separation decreases with $N$. However, there does not seem to be much to be gained in going to values of $N>256$, especially since the overhead of initialization and memory usage would then start to become significant.

With the possible exception of $N=10$ (which some may consider too small) all the generators shown in Fig. 3 satisfy all our criteria for highest quality RNG's with no known defects, provided of course that appropriate decimation is applied. This decimation can be read off the figure and is given in Table 1.

MIXMAX also offers the possibility to seed the generator at different faraway points in such a way as to avoid overlapping with sequences used in other calculations. So it satisfies all our criteria for highest quality as long as appropriate decimation is applied.

However, the developers of MIXMAX were already working on an extended MIXMAX which hopefully would not require any decimation.

\section{The High-Quality RNG's: 3. Extended MIXMAX}

The search for a generating matrix with larger entropy (for faster convergence to asymptotic mixing) consists in looking for transformations of $A$ which do not change the determinant, and do not interfere with the algorithm for fast multiplication but do move the eigenvalues further away from the unit circle. For the purposes of this section, it will be convenient to rewrite Eq. (1) as it is implemented in the computer algorithm, using explicitly integer arithmetic:

$a(i+1)=A \times a(i) \bmod p$,

where $a(i)$ is now an $N$-vector of 60-bit integers and $p=\left(2^{61}-1\right)$, so the real $x$ of Eq. (1) are now approximated by the rational $a / p$. As before, $A$ is an $N \times N$ matrix of 


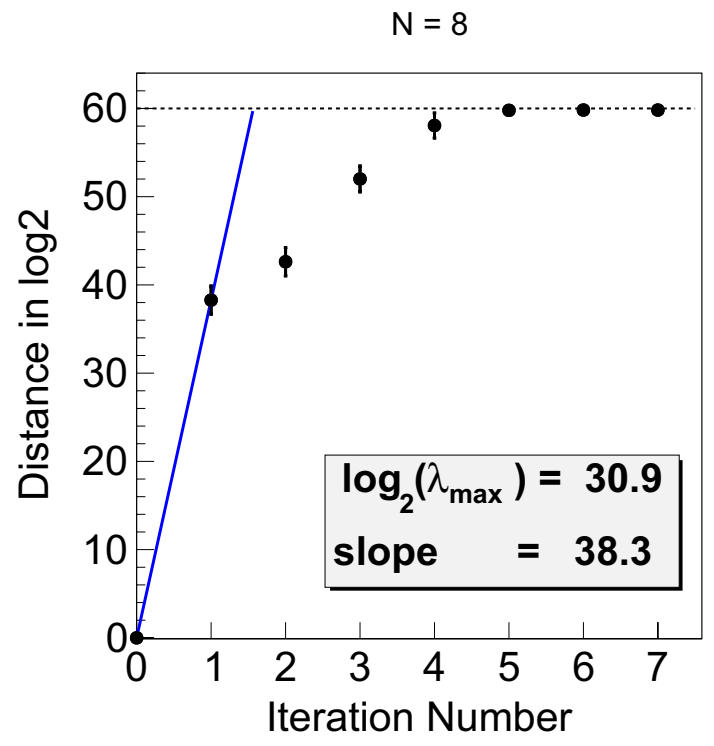

Fig. 4 Divergence of nearby trajectories for extended MIXMAX for $N=8, m \approx 2^{36}$

integers, but in extended MIXMAX, some of the integers may be very large.

The simplest modification which increases the entropy is to use much larger values of the magic integer $s$ in (3) up to the largest integers allowed by the computer arithmetic. This is called in [17] the two-parameter family $A(N, s)$ with large $s$.

A further modification was introduced using a new integer $m$ to produce a new matrix $A$ which is called the threeparameter family $A(N, s, m)(5)$, where it can be seen that for $m=1, A(N, s, m)$ reduces to the two-parameter $A$ :

$$
\begin{aligned}
& A(N, s, m) \\
& \quad=\left(\begin{array}{ccccccc}
1 & 1 & 1 & 1 & \cdots & 1 & 1 \\
1 & 2 & 1 & 1 & \cdots & 1 & 1 \\
1 & m+2+s & 2 & 1 & \cdots & 1 & 1 \\
1 & 2 m+2 & m+2 & 2 & \cdots & 1 & 1 \\
1 & 3 m+2 & 2 m+2 & m+2 & \cdots & 1 & 1 \\
\vdots & \vdots & \vdots & \vdots & \ddots & \vdots & \vdots \\
1 & (N-2) m+2 & (N-3) m+2 & (N-4) m+2 & \cdots & m+2 & 2
\end{array}\right)
\end{aligned}
$$

The main properties of the new generator were published in [17] for selected values of $(N, s, m)$ for which the period could be determined and which would be of most interest. Some of the results were nothing short of spectacular.

Probably the most impressive was the spectrum of eigenvalues for the parameter values: $N=240, m=2^{51}+1$, and $s=487013230256099140$, for which all the eigenvalues have modulus around $10^{15}$ except for one which is so close to zero that the product of all of them is one, as it must be.

However, as was the case for the original MIXMAX, the authors did not publish any results concerning the divergence

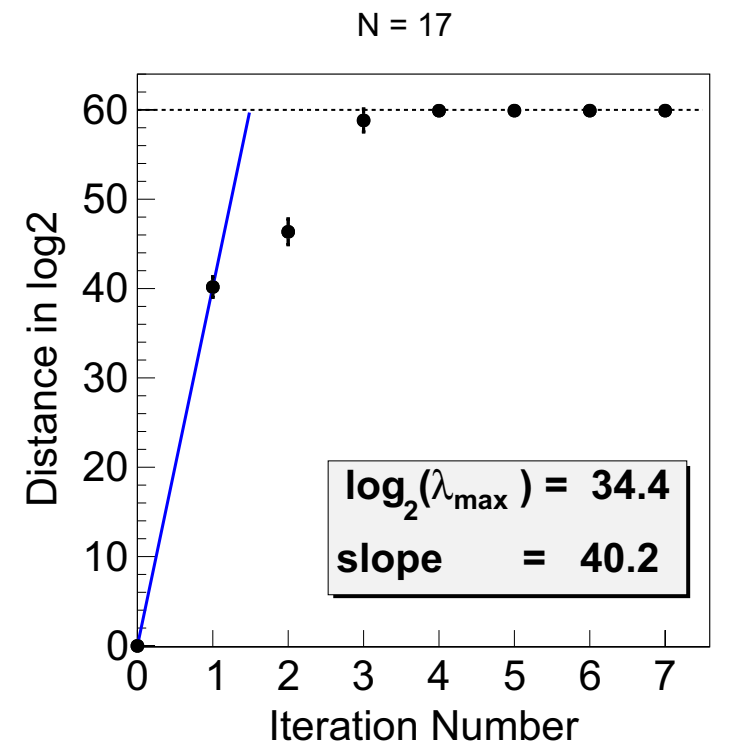

Fig. 5 Divergence of nearby trajectories for extended MIXMAX for $N=17, m \approx 2^{36}$

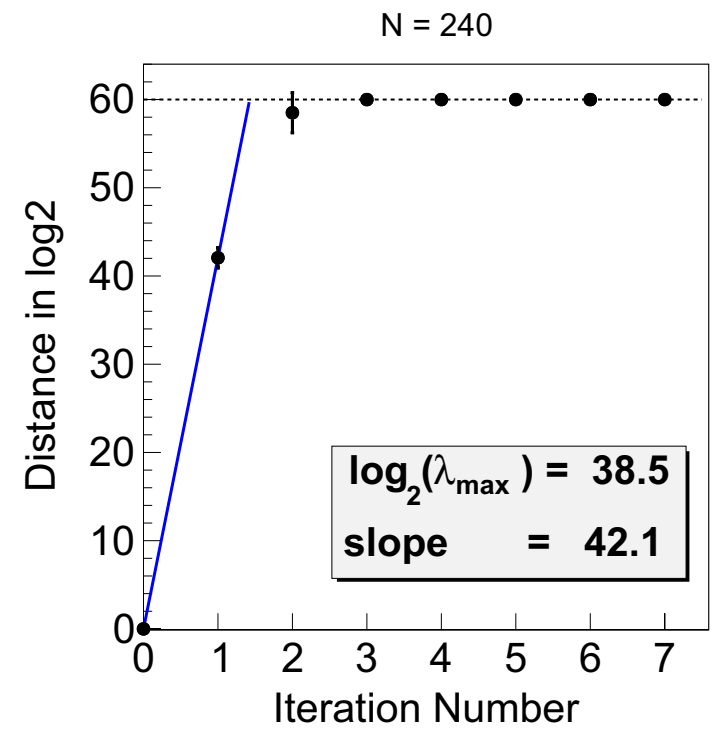

Fig. 6 Divergence of nearby trajectories for extended MIXMAX for $N=240, m \approx 2^{32}$

of nearby trajectories for these generators. So we calculated this ourselves, and the results are shown in Figs. 4, 5, and 6 for three interesting values of the parameters $(N, s, m)$ which are recommended by the MIXMAX developers in [18].

It is clear from these figures that none of these extended MIXMAX generators exhibit exponential divergence as they should for a K-system and as they do for original MIXMAX. Furthermore, none of the "slopes" that one could identify on these curves corresponds to the known 


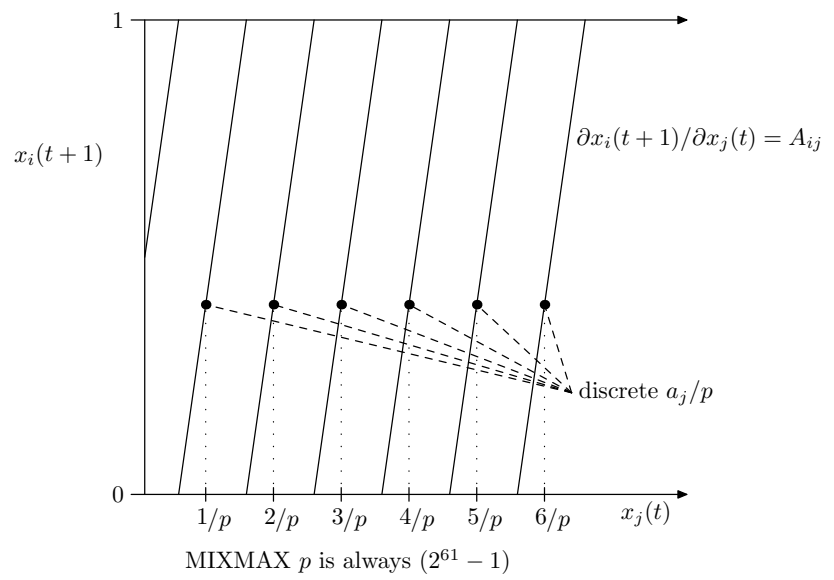

Fig. 7 Unwrapped torus for the case $A_{i j}=p$ with the continuous function: $x_{i}(t+1)=\sum_{j} A_{i j} x_{j}(t) \bmod 1$ and the discrete points $a / p$ of Eq. 4

Lyapunov exponent as was the case for the original MIXMAX. The same plots for extended MIXMAX for even larger values of $m$ (but still keeping $N m<p$ ), show a divergence so fast that it reaches the asymptotic value on the first step, so it is impossible to see whether the divergence is exponential.

We have tried to find the reason for this behaviour. The obvious suspect is the large values of some elements of $A$ which have caused the extended algorithm to lose the mixing properties of the continuous K-system. One upper limit on the allowed magnitude of elements of $A$ is given by the developers of MIXMAX in [17]:

It is most advantageous to take large values of $m$, but preferably keeping $N m<p$, such as to have an unambiguous correspondence between the continuous system and the discrete system on the rational sublattice.

The condition $\mathrm{Nm}<p$ is certainly necessary as indicated in the above quotation, but the values of $m N$ in our examples already satisfy that criterion, so there must be an additional explanation having to do with the multiplication of large integers (modulo $p$ ).

Let us look first at the continuous system of Eq. 1 and consider for example just one component of the matrixvector multiplication in the continuous system, that is, how the $i$ th component of the new vector $x(t+1)$ depends on the $j$ th component of the previous vector $x_{j}(t)$ :

$x_{i}(t+1)=A_{i j} x_{j}(t) \bmod 1$,

When the integer $A_{i j}$ is very big, this equation describes a function that loops rapidly around the torus, as indicated by the diagonal lines in Fig. 7. and when $A_{i j}$ is equal to $p$, there is only one discrete point on each loop of the continuous trajectory around the torus. Clearly one point is not even enough to "see" whether or not the continuous trajectory goes around the torus, but how many points on each turn would be required to represent faithfully the continuous trajectory?

For the complete trajectory in 240 dimensions it becomes difficult to determine what values of $A_{i j}$ are small enough. Fortunately we can again make use of the divergence of nearby trajectories, which must be exponential for the continuous system, so it should also be exponential for a discrete system which is a good approximation to the continuous system.

The lack of exponential divergence in Figs. 4, 5, and 6 means that extended MIXMAX with the given values of $N, m, s$ does not meet our criteria for K-mixing.

\section{Extended MIXMAX: The Spectral Test and Skipping}

Although originally the spectral test was applicable only to simple LCG's, it has been extended more recently to more complex LCG's, and L'Ecuyer et al. have applied it [19] to extended MIXMAX which can be analyzed as a matrix LCG. They find that the lattice structure in certain dimensions (notably in 5 dimensions for $N=240$ and $m=2^{51}+1$ ) is "very bad", all points lying on a number of hyperplanes much smaller than should be the case for their $p=2^{61}-1$. They also point out the existence of "simple linear relations" between coordinates of successive points which indicate unwanted correlations. Both problems remain even when the guilty coordinates are skipped in the output.

These problems were known to us but we did not consider them serious, because they were both eliminated (at least in RANLUX) by decimation as described above in this paper. Note that the skipping of coordinates performed by L'Ecuyer et. al. in [19] (and also by the MIXMAX developers in [18]) is very different from the decimation proposed in RANLUX. L'Ecuyer et. al. discard certain coordinates of every vector generated, whereas Lüscher discards whole vectors at a time. The latter is justified by the asymptotic nature of K-mixing so the fact that it is effective for RANLUX is further indication that K-mixing is indeed occurring. For extended MIXMAX, we cannot use the theory of K-mixing to justify decimation.

\section{The High-Quality RNG's: 4. RANLUX++}

As mentioned above in connection with the spectral test, the RANLUX algorithm is known to be equivalent to a linear congruential generator (LCG) with an enormous multiplier. This fact was used by Lüscher [3] to apply the spectral test to RANLUX, but otherwise seemed to be of little practical 
interest, since the computers of that time were unable to do long multiplications fast enough to be useful. More recently, however, Sibidanov [8] has produced a version called RANLUX++, implemented using the linear congruential algorithm with a clever way of doing long integer multiplication on modern computers, which makes it faster than standard RANLUX and does the decimation at the same time. The algorithm of Sibidanov cannot be programmed efficiently in the usual high-level languages, but is possible in assembler code using operations he says are now available on most computers used for scientific computing.

The heart of the new software is the procedure to multiply two 576-bit integers using 81 multiplications of $64 \times 64$ bits cleverly organised to be extremely fast using modern extensions of arithmetic and fetching operations described in Sibidanov [8]. For our purposes, there are two important features of this method: First, that it is able to reproduce the sequences of RANLUX much faster than the high-level language versions, and second, that it can produce more decimation (higher luxury levels) than the predefined levels in RANLUX, without increasing the generation time.

To understand the importance of this second feature, we should look more closely at the mechanism used to implement RANLUX with the LCG algorithm. Using the notation of Sibidanov, one random number $x_{i+1}$ is generated by

$x(i+1)=a \cdot x(i)+c \bmod m$.

With the parameters corresponding to RANLUX, the base $b=2^{24}$, the modulus $m=b^{24}-b^{10}+1$, the multiplier $a=m-(m-1) / b$, and $c=0$.

Decimation at level $p^{3}$ in RANLUX is implemented by delivering 24 numbers to the user, then throwing away $p-24$ numbers before delivering the next 24 . In RANLUX ++ , the decimation is called "skipping" [8], because it is implemented directly by

$x(i+p)=a^{p} \bmod m \cdot x(i) \bmod m$,

where $a^{p} \bmod m$ is a precomputed very long integer constant. As expected, this makes generation in RANLUX++ much faster than that of the original RANLUX. But the surprise comes when we look at the values of $a^{p}$ mod $m$ for different values of $p$ corresponding to the standard luxury levels, as well as two new higher levels, $p=1024$ and $p=2048$, shown in Table 1 of [8].

\footnotetext{
${ }^{3}$ Note that this $p$ is not related to the symbol $p$ used in the discrete approximation of $x$ by $a / p$. Since no confusion is possible, we prefer to use the same notation as the original authors of the algorithms.

${ }^{4}$ The values of $p$ corresponding to different luxury levels have changed, so the value used by Sibidanov (389) is slightly different from the value for the current highest luxury level for the 24-bit algorithm (397), but the difference is not significant.
}

Here, we see that as the luxury level increases, the obvious regularity in the factor $a^{p}$ mod $m$ decreases, but is still clearly present for the value of $p$ corresponding to the highest standard luxury level of RANLUX, $p=389 .{ }^{4}$ Sibidanov remarks that apparently, it would be advantageous to apply still more decimation, at least to $p=1024$ or $p=2048$, which can be obtained "for free". In this context, we have applied a simple test of randomness to the hex patterns, based on counting runs of identical hex digits, for the factors $a^{p} \bmod m$ for the two new values of $p$. We find that they both pass the test for expected numbers of 2-runs, 3 -runs and higher, which would indicate that the patterns are already as random for $p=1024$ as expected for a truly random sequence of 144 hex digits. So, if the randomness of $a^{p}$ mod $m$ is an indication of the degree of mixing, $p=389$ is not enough, but $p=1024$ is already fully mixing.

\section{Conclusions}

We now have several high-quality RNG's available for general use based on the theory of Mixing in classical dynamical systems.

\section{RANLUX}

This easily portable and well-documented RNG exists in both single- and double-precision versions both of which satisfy our criteria for K-mixing. At the highest standard luxury level (currently level 2, $p=397$ for the single-precision version, $p=794$ for the double-precision version), it has for many years been considered the most reliable RNG available, but too slow for some applications. Recent improvements in code optimization have speeded it up considerably, so it should now be acceptable for most applications (see timings below).

However, new evidence from RANLUX++ indicates that even more decimation up to $p=1024$ may be needed to eliminate the higher-order correlations. This level of decimation would make RANLUX slower, but is obtained "for free" with RANLUX++.

\section{MIXMAX}

We consider only the original MIXMAX with small $s$ and no $m$, described in [15]. This RNG produces always double-precision floating-point numbers, and the user has the freedom to choose $N$, the size of the generating matrix $A$. In practice one will choose a value of $N$ for which the period is known, tabulated in [15]. The code is maintained and available under HepForge.

For users who require the highest quality random numbers, it will be necessary to introduce into MIXMAX the 
possibility to decimate the appropriate number of iterations for each vector of random numbers delivered to the user, which is not difficult but requires modifying the source code. This has already been done for the version with $N=256$ installed at CERN. The decimation required for other values of $N$ is given here in Table 1.

Assuming the user has chosen a value of $N$ and has introduced appropriate decimation according to the criteria given here for RANLUX, he or she is still faced with the problem whether additional decimation may be useful to eliminate higher order correlations which have never been observed. But this "problem" could actually turn out to be an advantage in case of doubt whether the RNG is good enough for the application at hand: the calculation can be repeated with even higher degree of decimation and if the result is significantly different, we can use the higher degree. The theory guarantees that the higher decimation should only improve the mixing if it is not yet complete.

\section{RANLUX++}

This reincarnation of RANLUX by Alexei Sibidanov looks like it is going to be hard to beat, since it is the LCG equivalent of the RANLUX algorithm with even more decimation and runs faster than any other RNG considered here. Although we have as yet little experience using it, the only drawback we can see is that parts of it cannot be coded in the usual high-level languages, because it requires access to arithmetic instructions available only in assembler code. But the author claims these instructions are available on all the computers commonly used for MC calculations, so that the individual physicist should be able to obtain a copy that will run on his/her machine.

\section{Timing Benchmarks}

We think that the overriding consideration in choosing a RNG should be the quality of the random numbers to reduce to a minimum the risk of obtaining an incorrect result which may never be recognised as such. However, since some applications may require in addition a high speed of generation, we give some typical timings we have observed for the RNG's described here which meet our criteria for highest quality and no known defects. That means for RANLUX the highest standard luxury level and for MIXMAX 256 decimation "skip 5". For RANLUX++ the decimation is even much higher, since it is obtained "for free" as described above.

The table below gives our timings in nanoseconds per random floating-point number between zero and one observed on three different platforms A, B, C.

\begin{tabular}{lllll}
\hline Single Prec. & & A & B & C \\
& RANLUX S & 17.6 & 18.8 & 36.4 \\
& RANLUX++ & n.a. & 5.9 & 10.2 \\
Double Prec. & & & & \\
& MIXMAX 256 & 17.0 & 18.2 & 31.3 \\
& RANLUX D & 31.4 & 34.4 & 67.5 \\
& RANLUX++ & n.a. & 7.9 & 13.7 \\
\hline
\end{tabular}

Platform A is a MacBook i7, $2.6 \mathrm{GHz}$; B is a desktop i9-9900K, 3.7 GHz; and C is a server Xeon E5-2683, $2 \mathrm{GHz}$. RANLUX++ did not run on our MacBook. RANLUX is often used with SSE extensions which are foreseen in the standard code and speed it up by about $30 \%$ but are not portable.

Acknowledgements We have benefitted greatly from discussions over many years with Martin Lüscher and George Savvidy, and we are also grateful for contributions from Konstantin Savvidy, John Harvey, John Apostolakis, and Philippe De Forcrand.

Open Access This article is licensed under a Creative Commons Attribution 4.0 International License, which permits use, sharing, adaptation, distribution and reproduction in any medium or format, as long as you give appropriate credit to the original author(s) and the source, provide a link to the Creative Commons licence, and indicate if changes were made. The images or other third party material in this article are included in the article's Creative Commons licence, unless indicated otherwise in a credit line to the material. If material is not included in the article's Creative Commons licence and your intended use is not permitted by statutory regulation or exceeds the permitted use, you will need to obtain permission directly from the copyright holder. To view a copy of this licence, visit http://creativecommons.org/licenses/by/4.0/.

\section{References}

1. Knuth DE (1998) The art of computer programming, volume 2: semi-numerical algorithms, 3rd edn. Addison-Wesley, Reading

2. Ferrenberg AM, Landau DP, Wong YJ (1992) Monte Carlo simulations: Hidden errors from "good" random number generators. Phys Rev Lett 69:3382

3. Lüscher M (1994) A portable high-quality random number generator for lattice field theory simulations. Comput Phys Commun 79:100

4. Arnold VI, Avez A (1989) Ergodic problems of classical mechanics. Addison-Wesley, Redwood

5. Savvidy G, Ter-Arutyunyan-Savvidy N (1991) On the Monte Carlo simulation of physical systems. J Comput Phys 97:566

6. Savvidy G (2016) Anosov C-systems and random number generators, theoretical and mathematical physics, arXiv:1507.06348

7. Berkovitz J, Frigg R, Kronz F (2006) The ergodic hierarchy, randomness and Hamiltonian chaos. Stud Hist Philos Mod Phys 37:661-691

8. Sibidanov A (2017) A revision of the subtract-with-borrow random number generators. Comput Phys Commun 221:299-303

9. Maclaren NM (1992) A limit on the usable length of a pseudorandom sequence. J Stat Comput Simul 42:47-54

10. Marsaglia G, Zaman A (1991) A new class of random number generators. Ann Appl Prob 1:462

11. Tezuka S, L'Ecuyer P, Couture R (1993) On the lattice structure of the add-with-carry and subtract-with-borrow random number generators. ACM Trans Model Comput Simul 3(4):315-331 
12. James F (1994) RANLUX: a Fortran implementation of the highquality pseudorandom number generator of Lüscher. Comput Phys Commun 79:111

13. Hamilton KG, James F (1997) Acceleration of RANLUX. Comput Phys Commun 101:241-248

14. Shchur LN, Butera P (1998) The RANLUX generator: resonances in a random walk test. Int J Mod Phys C 9:607-624 arXiv:heplat/9805017

15. Savvidy K (2015) The MIXMAX random number generator. Comput Phys Commun 196:161-165. https://doi.org/10.1016/j. cpc.2015.06.003. arXiv:1404.5355

16. L'Ecuyer P, Simard R (2007) TestU01: a software library in ANSI $\mathrm{C}$ for empirical testing of random number generators. ACM Trans Math Softw 33:22
17. Savvidy K, Savvidy G (2016) Spectrum and entropy of C-systems. MIXMAX random number generator. Chaos Fract Solitons 91:33; e-print arXiv: 1510.06274

18. Martirosyan N, Savvidy K, Savvidy G (2019) Spectral test of the MIXMAX random number generators. arXiv: $1806.05243 \mathrm{v} 2$

19. L'Ecuyer P, Wambergue P, Bourceret E (2017) Spectral analysis of the MIXMAX random number generators. Submitted to INFORMS J Comput

Publisher's Note Springer Nature remains neutral with regard to jurisdictional claims in published maps and institutional affiliations. 